MATEC Web of Conferences 41, 02002 (2016)

DOI: $10.1051 /$ matecconf/20164102002

(C) Owned by the authors, published by EDP Sciences, 2016

\title{
Dynamic Optimization for Vortex Shedding Suppression
}

\author{
loannis Bonis ${ }^{1}$, Haralambos Sarimveis ${ }^{2}$ and Dimitrios Koubogiannis ${ }^{1, a}$ \\ ${ }^{1}$ Dept of Energy Technology Engineering, Technological Educational Institute of Athens, Agiou Spyridonos Street, \\ 12210 Egaleo, Athens, Greece. \\ ${ }^{2}$ School of Chemical Engineering, National Technical University of Athens, 9 Heroon Polytechniou Street, \\ 15780 Zografou, Athens, Greece.
}

\begin{abstract}
Flows around structures exhibiting vortex shedding induce vibrations that can potentially damage the structure. A way to avoid it is to suppress vortex shedding by controlling the wake. Wake control of laminar flow behind a rotating cylinder is formulated herein as a dynamic optimization problem. Angular cylinder speed is the manipulated variable that is adjusted to suppress vortex shedding by minimizing lift coefficient variation. The optimal angular speed is assumed to be periodic like wake formation. The control problem is solved for different time horizons $t_{\mathrm{H}}$. The impact of $\mathrm{t}_{\mathrm{H}}$ to control is evaluated and the need for feedback is assessed.
\end{abstract}

\section{Introduction}

Flow separation and vortex shedding are often exhibited in the built environment giving rise to vortex induced vibrations that can potentially lead to structure damage. Thus, it is important to control the phenomenon effectively. Both active and passive control techniques can be applied. A promising technique, also followed herein, is to control boundary layer and wake by use of rotating cylinders [1].

The proposed method is based on a detailed transient Computational Fluid Dynamics (CFD) model for the numerical simulation of the flow around a $2 \mathrm{D}$ cylinder exhibiting laminar vortex shedding. This model has been recently validated [2]. For a given Reynolds number, the appropriate cylinder rotation strategy in order to achieve vortex shedding minimization is calculated by solving an optimal control problem. This problem is formulated in terms of a suitable objective function and using the transient CFD model as a constraint. The arising dynamic optimization problem is solved by means of a feasible path method. Cylinder angular velocity is considered to follow sinusoidal law in time described by three parameters (amplitude, frequency and phase) which are the independent variables of the optimization problem.

In what follows, the formulation of the control problem is presented, its solution is validated in a benchmark test case, the effect of the time horizon $t_{H}$ on which control is based is evaluated and the need for feedback is assessed.

\section{Test case description}

The two-dimensional problem concerning the flow of a

\footnotetext{
${ }^{a}$ Corresponding author: dkoubog@teiath.gr
}

fluid with density $\rho$ past a stationary or rotating cylinder of diameter $D[1]$ is used as a test case herein. The continuity and momentum governing equations (i.e. the Navier-Stokes equations), in dimensionless form are written as:

$$
\begin{gathered}
\nabla \cdot u=0 \\
\frac{\partial u}{\partial t}+(u \cdot \nabla) u=-\nabla p+\frac{1}{\operatorname{Re}} \nabla^{2} u
\end{gathered}
$$

The computational domain is shown in Figure 1. Reynolds $R e$ number is based on inflow velocity. The boundary conditions for (1) and (2) are $u=(1,0)$ on $\Gamma_{1}$, $p=0$ on $\Gamma_{3}, \quad \frac{\partial u_{x}}{\partial y}=0, u_{y}=0$ on $\Gamma_{2}, \Gamma_{4} \quad$ and $u=\left(\gamma_{x}, \gamma_{y}\right)$ on $\Gamma_{c}, \gamma$ being the angular velocity of the cylinder. The initial condition is $\left.u\right|_{t=0}=(0,0)$. Equations (1) and (2) are numerically solved by means of COMSOL Multiphysics software [3] on an unstructured mesh (of 4528 triangular elements and 20766 nodes). The Finite Element Method using Lagrange $\mathrm{P}^{2}-\mathrm{P}^{1}$ elements has been implemented. In the following, the discretized equations (1) and (2) with their boundary conditions will be denoted by $f_{N S-c}=0$. 


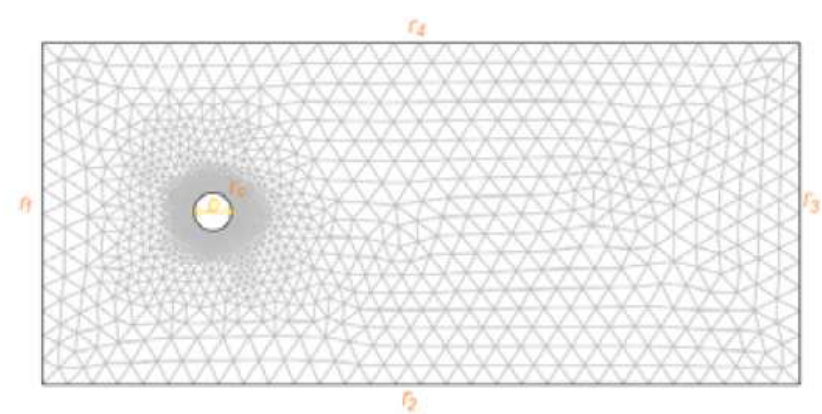

Figure 1. Computational domain and computational mesh for the benchmark stationary cylinder problem.

Important parameters of such a flow system are the Reynolds number $(R e)$ and cylinder angular velocity $(\gamma)$. Here, laminar flow of $R e=100$ with $\gamma=0$, which exhibits vortex shedding and turns to be periodic, was simulated and validated against literature data [1]. Figure 2 shows the time evolution of the cylinder lift $\left(c_{L}\right)$ and drag $\left(c_{D}\right)$ coefficients. From this figure it seems that a period of $T=5.5$ non-dimensional time units characterizes the phenomenon.

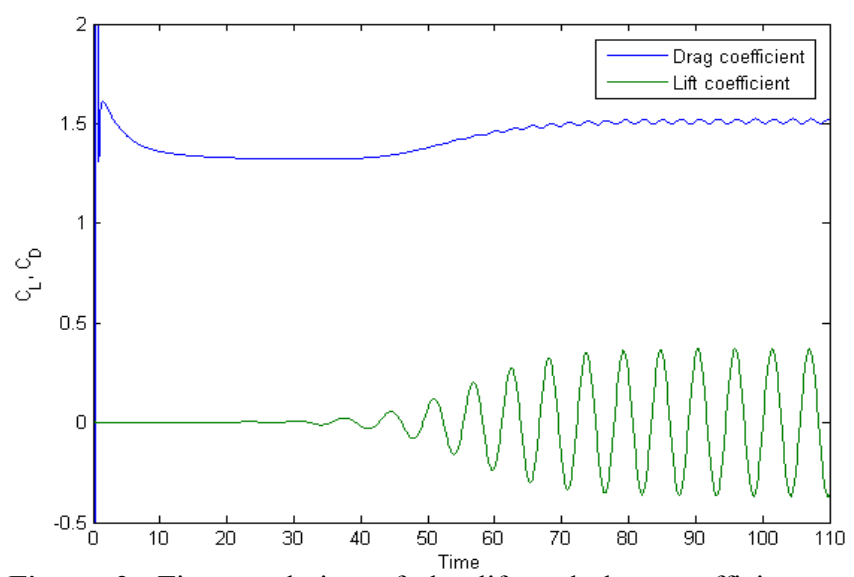

Figure 2. Time evolution of the lift and drag coefficients computed by the CFD model for the benchmark stationary cylinder problem of $R e=100$.

\section{Methodology}

Aiming to minimize wake formation by means of minimizing lift coefficient variation, an appropriate dynamic optimization problem is formulated in order to identify the optimal strategy for the angular cylinder velocity $\gamma$ (manipulated variable), as follows

$$
z_{\text {opt }}=\arg \min _{z}\left\{f\left(z ; t_{H}\right)\right\}
$$

where

$$
f\left(z ; t_{H}\right)=\int_{t_{\text {in }}}^{t_{\text {in }}+t_{H}}\left[c_{L}\left(\gamma\left(z, t ; t_{H}\right), u, t\right)\right]^{2} d t
$$

subject to

$$
\begin{gathered}
\gamma\left(z, t ; t_{H}\right)=z_{1} \sin \left(z_{2} t+z_{3}\right) \\
f_{N S-c}\left(\gamma\left(z, t ; t_{H}\right), u, t\right)=0
\end{gathered}
$$

phenomenon, a periodic expression (5) has also been considered for the control variable $\gamma$. The latter has been parameterized by a sinusoidal expression in time, $\gamma\left(z, t ; t_{H}\right)=z_{1} \sin \left(z_{2} t+z_{3}\right)$, employing a vector of three independent variables $z=\left[z_{1}, z_{2}, z_{3}\right]^{T}$ (which correspond to the magnitude, the frequency and the phase of the sinusoidal control action). The time horizon $t_{H}$ used to solve the optimization problem is set herein to be a multiple of the period $T$ that characterizes the uncontrolled (stationary) cylinder vortex shedding.

The application of a control action to a periodic system perturbs it and alters its periodicity. For the optimization problem examined herein, the cylinder is initially considered stationary and control is applied after the system reaches a periodic state. Thus, the system is simulated with $\gamma=0$ and zero initial conditions for adequate time and after periodicity has been established, the control is applied at time instance $t_{i n}$. A value of $t_{i n}=100$ non-dimensional time units was used herein, based on the evolution of the lift coefficient shown in Figure 2. As an initial condition for the optimal control problem, the state variables (velocity and pressure) corresponding to $\gamma=0$ and $t=t_{i}$ have been considered.

The objective function $f\left(x ; t_{H}\right)$ given by (4) corresponds to the area below $c_{L}^{2}$-curve for time interval $t_{H}$. By minimizing this, the minimization of $c_{L}$ variation is sought and accordingly the suppression of vortex shedding. The optimization problem is solved by the MATLAB implementation of active-set deterministic optimization [4]. The independent variables are bounded so that $z_{1}>0,-\pi / 2 \leq z_{2} \leq \pi / 2$ and $0 \leq z_{3} \leq T / 2$. The computing cost is accounted in terms of function evaluations rather than actual computational time. Most of this time is expended on the solution of the constraint, namely the CFD problem.

\section{Results and discussion}

The optimal control problem in equation (3) aims to minimize variation of $c_{L}$ in time $t_{H}$ after enabling the controller. Three dynamic optimization scenarios have been established, using as many discrete values for $t_{H}$, namely $T, 2 T$ and $5 T$. For each $t_{H}$, a different rotation strategy

$$
\gamma_{t_{H}}=\gamma\left(z_{\text {opt }}, t ; t_{H}\right)=z_{1, o p t} \sin \left(z_{2, o p t} t+z_{3, o p t}\right)
$$

was computed by solving the corresponding mathematical programming problem. For each of the calculated strategies $\left(z_{\text {opt }}\right)$, three evaluations of the objective function were performed by

$$
f_{t_{f}}=f\left(z_{\text {opt }} ; t_{f}\right)=\int_{t_{i n}}^{t_{i n}+t_{f}}\left[c_{L}\left(\gamma\left(z_{\text {opt }}, t ; t_{H}\right), u, t\right)\right]^{2} d t
$$

Since the wake formation is a periodic 
for $\left.\mathrm{t}_{\mathrm{f}}=\mathrm{T}, 2 \mathrm{~T}, 5 \mathrm{~T}\right)$. This means that, having set the control action to a particular $\gamma_{t_{H}}=\gamma\left(z_{\text {opt }}, t ; t_{H}\right)$, the objective $f_{t_{f}}=f\left(z_{\text {opt }} ; t_{f}\right)$ was computed for different intervals $\mathbf{t}_{\mathrm{f}}$ under the action of the same controller. Table 1 summarizes the results.

At each line of Table 1, the corresponding objective $f_{t_{f}}=f\left(z_{\text {opt }} ; t_{f}\right)$ becomes minimum for $t_{f}=t_{H}$, i.e. $\min f\left(z_{\text {opt }} ; t_{f}\right)=f\left(z_{\text {opt }} ; t_{H}\right)$ (Table 1 , cells in grey). This is expected, since the control horizon in the optimization problem was $t_{H}$. This fact suggests that the controller works correctly, at least qualitatively.

According to the same Table 1 , the lower the $t_{H}$, the greater the $f_{t_{f}}$ value for greater $t_{f}$. This means that the objective function exhibits a greater deviation from both controlled and uncontrolled cases in the long-term when it has been optimized for small time horizon.

The final and most important remark is that percentage reduction in $f_{t_{H}}$ becomes significantly greater for smaller values of $t_{H}$. This reduction is a measure of control benefits, since it expresses the vortex suppression of the optimally controlled cylinder versus the corresponding value of the uncontrolled system with stationary cylinder. For larger control horizons, the benefits of optimal control diminish. Thus, it is more advantageous to perform control using small "bursts" than relying on control actions of larger duration. Hence, control formulations using finite control horizon, such as Model Predictive Control [5], seem more promising than optimization based on a long or infinite time horizon. In addition, shorter control horizons may incur the need for feedback, to avoid accumulation of approximation errors, which will lead to insufficient closed-loop performance.

Table 1. Objective function calculation for different time intervals $t_{f}$

\begin{tabular}{|c|c|c|c|c|c|}
\hline & $\gamma=0$ & $\gamma_{T}$ & $\gamma_{2 T}$ & $\gamma_{5 T}$ & $\begin{array}{c}\text { Reduction in } \\
f_{t_{H}}\end{array}$ \\
\hline$f_{T}$ & 3.22 & 1.83 & 2.49 & 2.84 & $43.2 \%$ \\
\hline$f_{2 T}$ & 6.64 & 23.80 & 6.11 & 6.26 & $7.98 \%$ \\
\hline$f_{5 T}$ & 16.96 & 461.4 & 18.80 & 16.78 & $1.06 \%$ \\
\hline
\end{tabular}

The values of the optimal control variables that were computed for the time horizons $\mathrm{T}, 2 \mathrm{~T}$ and $5 \mathrm{~T}$ are

$z_{\text {opt }, T}=[0.791,-0.065,0.207]^{T}$

$z_{o p t, 2 T}=[1.012,-0.004,0.048]^{T}$

$z_{\text {opt }, 5 T}=[0.967,-0.0006,0.018]^{T}$

For their calculation 84, 71 and 76 function evaluations were required, respectively. Each function evaluation is a full CFD simulation using COMSOL, for a simulation time equal to $t_{H}$. Looking at the optimum values above, it can be noticed that $z_{\text {lopt }}$ is of the same order of magnitude in all cases. Concerning $z_{2 o p t}$, it decreases as the control horizon increases and weakens the impact of time $t$ on the variation of $\gamma$, since the term $z_{2 o p t} t$ in $\gamma\left(z_{\text {opt }}, t ; t_{H}\right)=z_{\text {lopt }} \sin \left(z_{2 o p t} t+z_{3 o p t}\right) \quad$ becomes small. Figure 3 shows the variation of $\gamma$ in time. The control function has a large period and the larger the optimization horizon $t_{H}$, the larger this period is (Figure 4).

Figure 5 illustrates the temporal evolution of the lift coefficient for the uncontrolled system, as well as for the controlled system using the three optimal cylinder rotation strategies. This graph contains the data used for the calculation of the objective functions for the dynamic optimisation problems formulated. $\mathrm{t}=100$ is the point when the system starts being controlled. It is evident that if we are only concerned with $1 T$, the green curve is best, which corresponds to the optimization problem with $t_{H}=1 T$, as it is closer to 0 . This is a visual verification of the remarks we made using Table $I$, in which aggregate values are used rather than time profiles.

Fig. 6 illustrates the corresponding temporal profile for the drag coefficient. Similarly, Table II presents the aggregate results for the cases presented in Table I, but here all calculations are based on the drag coefficient, rather than on the $c_{L}$. An expression similar to the objective function (4) has been used:

$$
g_{t_{f}}=\int_{t_{i n}}^{t_{i n}+t_{f}}\left[c_{D}\left(\gamma\left(z_{\text {opt }}, t ; t_{H}\right), u, t\right)\right]^{2} d t
$$

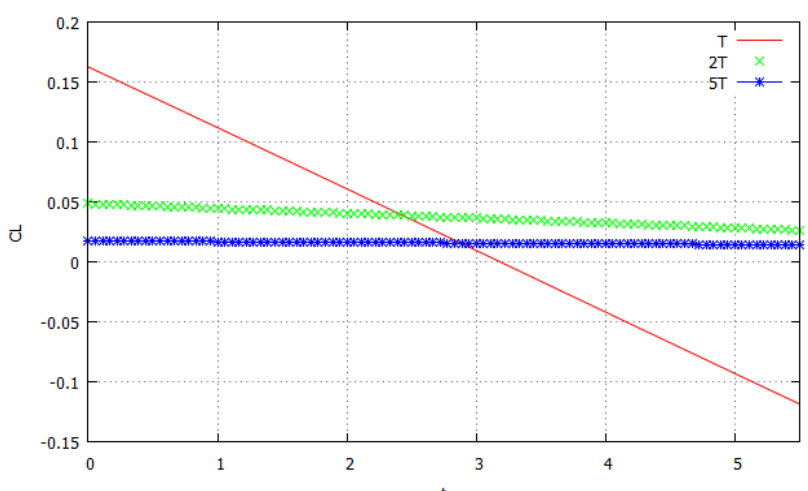

Figure 3. Variation of angular cylinder velocity $\gamma$ for various $t_{H}$ at time $\mathrm{T}$.

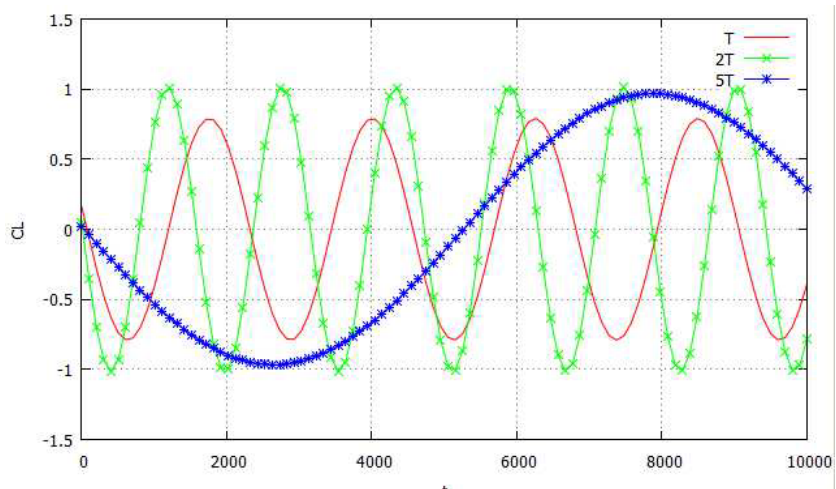

Figure 4. Variation of angular cylinder velocity $\gamma$ for various $t_{H}$ 
at the long-term.

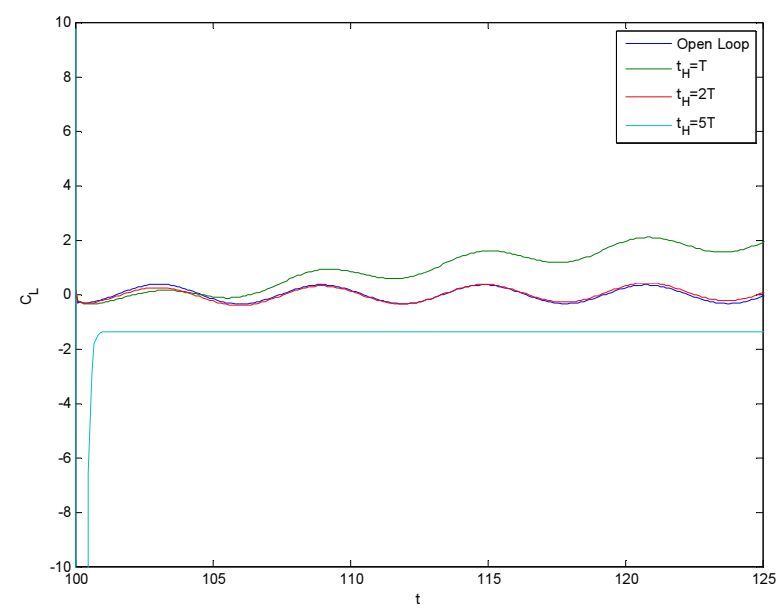

Figure 5. Time evolution of the lift coefficient employing the optimal cylinder movement strategies identified, as well as the case of stationary cylinder.

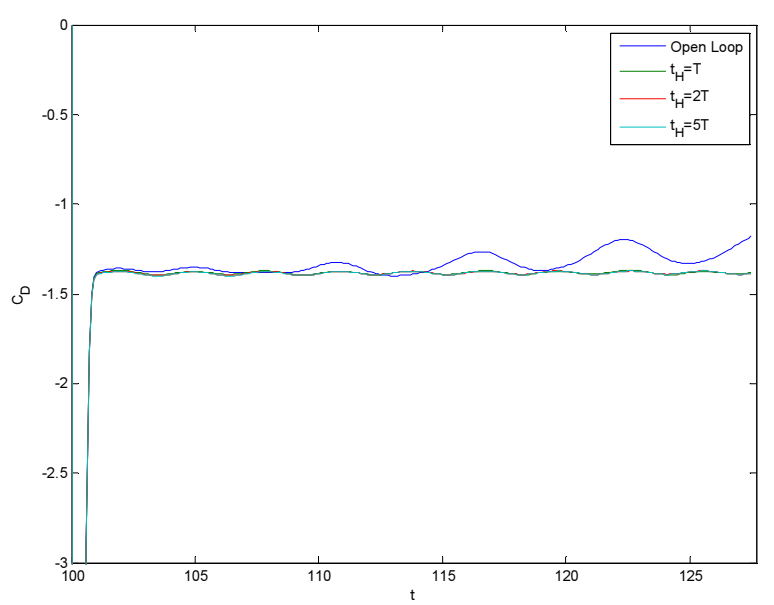

Figure 6. Time evolution of the drag coefficient employing the optimal cylinder movent strategies identified, as well as the case of stationary cylinder.

Table 2. Calculation of function (9) for different intervals $t_{f}$.

\begin{tabular}{|c|c|c|c|c|}
\hline $\begin{array}{c}\text { all values } \\
* 10^{5}\end{array}$ & $\gamma=0$ & $\gamma_{T}$ & $\gamma_{2 T}$ & $\gamma_{5 T}$ \\
\hline$g_{T}$ & 1.640577 & $\mathbf{1 . 6 4 0 0 9 2}$ & 1.640401 & 1.640511 \\
\hline$g_{2 T}$ & 1.641636 & $\mathbf{1 . 6 4 1 1 2 1}$ & 1.641454 & 1.641568 \\
\hline$g_{5 T}$ & 1.644792 & $\mathbf{1 . 6 4 3 9 6 0}$ & 1.644607 & 1.644723 \\
\hline
\end{tabular}

Although optimization was only performed on the merit of the lift coefficient, it was performed so that vortex shedding overall would be minimized and therefore the drag coefficient would be reduced as well. Table 2 illustrates that indeed the controlled system exhibits drag coefficient less than the uncontrolled one.
In particular, the values of the aggregate function of (9) are less for all control cases, in comparison to the uncontrolled case. However, the minima for the aggregate functions of the lift and drag coefficients (objective function (4) and equation (9), respectively) do not coincide. The greyed-out records of Table 2 indicate the position that the minimum value for the lift coefficient aggregate was found; the minimum for the drag coefficient aggregate is indicated in bold. This observation highlights the criticality of proper objective function selection while attempting to diminish vortex shedding using dynamic optimization.

\section{Concluding remarks}

A framework was presented for the optimal control of vortex shedding behind a cylinder. In a case concerning laminar flow around a rotating cylinder, its angular speed was the manipulated variable to minimize vortex shedding. Since wake formation is periodic, the optimal angular speed was considered to be sinusoidal. The control method worked satisfactorily. Parametric studies showed that it is more advantageous to perform control using small "bursts" rather than relying on control actions derived from considering long time horizons.

Further work concerns the effect of Reynolds number to the optimal control, the adoption of reduced order modeling techniques or surrogate models for the substitution of the full CFD model while preserving most of its accuracy to save computing time and the implementation of MPC instead of optimal control.

\section{References}

[1] W.R. Graham, J. Peraire, K.Y. Tang, Int. J. for Num. Meth. in Eng. 44, 945 (1999)

[2] D.G. Koubogiannis, H. Sarimveis, I. Bonis, FLOW 2014: 9th Panhellenic Conference on Fluid Transport Phenomena, 12-13 December, AthensGreece (2014)

[3] Comsol AB., COMSOL Multiphysics, 3.5 User's Guide (2008)

[4] Mathworks Inc., Matlab Optimization Toolbox User's Guide. [Online]. Available: http://www. mathworks.com/access/helpdesk/help/pdf_doc/optim /optim_tb.pdf (2007)

[5] E.F. Camacho, C.B. Alba, Model predictive control, Springer Science \& Business Media (2013) 